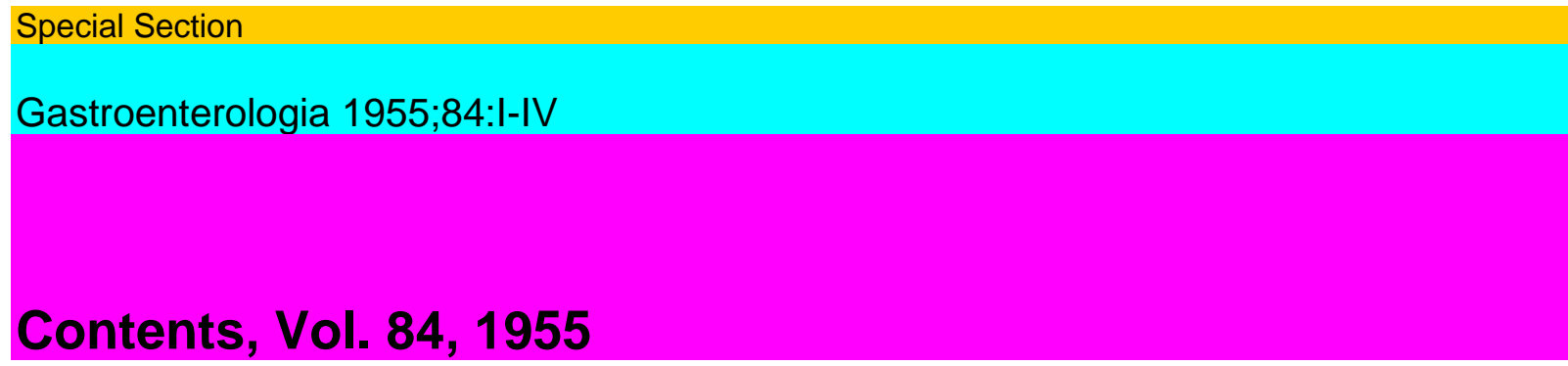

\title{
INDEX
}

Albot, G., A. Busson, J. Toulet und G. Bonnet, Röntgenuntersuchung der Gallenblase in 2 Richtungsebenen (A.P. und Profil) mit Beurteilung der beschleunigten Entleerungsprobe in bestimmten Zeitabständen («Cholécystographie F.P.A.») 143

Bajusz, E., vide M. Békés

Baur, II., Klinische Bedeutung der Lírokathepsinbestimmung. 2. Mitteilung 283

Békés, M., und E. Bajusz, Vitale tierexperimentelle Dehydrogenase-Enzymaktivitätsuntersuchungen an Magen mit peroralcr TTC-Verabreichung 164 Bonnet, G., vide G. Albot Busson, A., vide G. Albot Celio, A., Pathologisch-anatomische und klinische Erfahrungen mit der stenosierenden Diverticulitis coli unter besonderer Berücksichtigung ihrer Therapie $\quad 315$

Decker, P., et F. Saegesser, Résultats éloignés du traitemciit cliirurgical par exérèse du cancer de Гæsophage et du cardia 292

Demole, M., Données numériques sur la côlite ulcéreuse en Suisse 324

- $\quad$ et P. Rentchnick, Facteurs pathogéniques de la tuberculose des gastrectomisés $\quad 17$

Deucher, F., und K. Mülly, Unsere Erfahrungen mit der antethorakalen Ösophagusplastik $\quad 301$

Fellmann, N., Verdoppelung von. Colon und terminalem Ileum 148

Forell, M. M, Zur Frage des Entstehungsmechanismus des Kreislaufkollapses

bei der akuten Pankreasnekrose 225

Frank,A., Über den diagnostischen Wert der Magenbiopsie 1

Gerner, G., und N. Ilenning, Das Vitamin-B12-Bindungsvermögen des

menschlichen Magensaftes in Beziehung zum histologischen Bild der Fundusschleimhaut 103, 273

Gräsbeck, R., Fractionation of Human Gastric Juice and Saliva Employing Starch Electrophoresis 99

Hafter, E., Gastrointestinale Polypose mit Melanose der Lippen- und Mundschleimhaut (Peutz-Jeghers'sches Syndrom) 341

Henning, N., vide G. Gerner

Hess, W., Das Risiko abdomineller Eingriffe bei alten Patienten

Kalk, II., Leberfunktionsproben und bioptischer Befund 349

- Über die posthepatitische Hyperbilirubinämie 207

Kríeg, P., vide M. Mamie

Mamie, M., et P. Kríeg, Contribution à la casuistique des accidents de Гantibiothérapie 334 
Mülly, K., vide F. Deucher

Norpoth, L., Das «Pulvis stomachicus Birckmanm» aus dem 16. Jahrhundert. Ein Beitrag zur Geschichte der Magentherapie 169

Rentchnick, P., vide M. Demote

Saegesser, F., vide P. Decker

Schmidt-Kessen, W., Eine Methode zur Druckmessung im Magen-Darm-

\section{lumen 252}

Studer, H., Röntgendiagnostik der Gallenwege mit «Biligrafin»»

Toulet, J., vide G. Albot

Walk, L., Erosive Gastritis. Clinical Review and Analysis of Twenty-Seven

Cases 87

Übersichtsreferate - Surveys - Revues

Friedrich, L, Behandlung der Magenblutung

Strehler, E., Gegenwärtiger Stand der sog. «Vitamin-Li »-Tlierapie der

Magendarm-Ulcera

Geßellschaftsberichte - Society Transactions - Sociétés

XIXe Congrès de VAssociation d'études physiopathologiques dufoie. 2e session, Vichy, 17 et 18 septembre 1955 195

Sociêté Suísse de Gastro-entérologíe. Seance annuelle. Bale, 12 et 13 novembre 1955 372

Editorial

Azotemia in Gastro-intestinal Haemorrhages

La dyspepsie achlorhydrique

133

La «niche» radiologique, signe de cicatrisation de $\Gamma$ ulcère

Milk-Alkali-Syndrom 259

Seite der Therapie - Page de thérapeutique

$72,134,200,260$

Referate - Abstracts - Analyses

I. Allgemeines - Généralítés

II. Oesophagus

III. Magen - Estomac

Ulcus ventriculi

Carcinoma ventriculi

79

Gastrectomie $\quad 84$

Gastritis $\quad 84$

Diverticulum $\quad 86$

Nachtrag $\quad 136$

IV. Duodenum

V. Jejunum und Ileum

VI. Colon

VII. Appendix

140

VIII. Leber - Foie

Pathologische Physiologie

Hepatitis

Cirrhose 205, 262

IX. Gallenwege - Voies bilíaíres

X. Pankreas 264 
XL Varia

268

Buchbesprechungen - Book Reviews - Livres Nouveaux . . 86, 141, 206, 271, 379

Varia 86, 142, 272, 382

Personalia

272 